\author{
А.А. Костылев, Н.В. Пизова, Н.А. Пизов \\ ГБОУ ВПО «Ярославская государственная медицинская академия», Ярославль
}

\title{
Когнитивные нарушения при эпилепсии
}

Когнитивные нарушения при эпилепсии - важная проблема неврологии. В основе представления о патогенезе нарушений высших нервных функций лежит взаимодействие нескольких факторов, к которым относятся форма и длительность заболевания, гендерные различия, влияние противоэпилептической терапии. Обсуждается роль межприступных эпилептиформных изменений в формировании когнитивного дефицита у взрослых и эпилептических энцефалопатий у детей. Современные нейрофизиологические и нейровизуализационные методы диагностики позволяют выявлять новые особенности течения и прогрессирования нарушений функций высшей нервной деятельности при эпилепсии.

Ключевые слова: эпилепсия; когнитивные нарушения; противоэпилептическая терапия.

Контакты: Александр Анатольевич Костылев akostylev2010@gmail.com

Для ссылки: Костылев АА, Пизова НВ, Пизов НА. Когнитивные нарушения при эпилепсии. Неврология, нейропсихиатрия, психосоматика. 2013;(4):66-70.

\section{Cognitive impairments in epilepsy \\ A.A. Kostylev, N.V. Pizova, N.A. Pizov \\ Yaroslavl State Medical Academy, Yaroslavl}

Cognitive impairments in epilepsy are a current problem in neurology. The basis of the idea on the pathogenesis of higher nervous system dysfunctions is the interaction of a few factors that include the form and duration of the disease, gender differences, and the impact of antiepilep-

tic therapy. The role of interattack epileptiform changes in the development of cognitive deficit in adults and epileptic encephalopathies in children is discussed. Up-to-date neurophysiological and neuroimaging diagnostic methods allow the detection of new features in the course and progression of higher nervous system dysfunctions in epilepsy.

Key words: epilepsy; cognitive impairments; antiepileptic therapy.

Contact: Aleksandr Anatolyevich Kostylev akostylev2010@gmail.com

For reference: Kostylev AA, Pizova NV, Pizov NA. Cognitive impairments in epilepsy itis. Neurology, Neuropsychiatry, Psychosomatics. 2013;(4):66-70.

DOI: http://dx.doi.org/10.14412/2074-2711-2013-2458

Эпилепсия представляет собой хроническое полиэтиологическое заболевание, проявляющееся повторными неспровоцированными судорожными или другими припадками, нарушением сознания и изменениями личности $[1,2]$. В последнее время достигнут определенный прогресс в лечении эпилепсии [3], и наряду с достижением ремиссии также важными становятся изучение и коррекция неконвульсивных проявлений заболевания, к которым относятся изменения функций высшей нервной деятельности (ВНД) [4]. Во всем многообразии психической патологии выделяют собственно когнитивные нарушения, эпилептические психозы, изменения эмоционально-аффективной сферы, обсессивно-компульсивные расстройства, тревожные и панические состояния, эпилептические энцефалопатии [4-7].

Уже в начале изучения проблемы грубые психические изменения рассматривали как непременный атрибут заболевания. По наблюдениям французского психиатра Ж. Эскироля [1], нарушения психических функций у больных эпилепсией были разнообразными и имели тенденцию к утяжелению по мере прогрессирования заболевания. В 1860 г. Б. Морель предполагал наличие у больных эпилепсией «интеллектуальной ауры» как эпилептического эквивалента.
Выделение отдельных функций коры больших полушарий и теория Д.Х. Джексона об иерархичной структуре нервной системы способствовали в XIX в. дальнейшему изучению структурных изменений и их роли в развитии симптомов эпилепсии. Характерные изменения гиппокампа, названные В. Зоммером (1880) гиппокампальным склерозом, начали ассоциировать с развитием деменции при эпилепсии. Однако этими находками не удалось полностью объяснить разнообразие когнитивных нарушений. Первые психодиагностические исследования были выполнены в конце XIX начале XX в. и связаны с именами В.М. Бехтерева, Э. Крепелина, А.Н. Бернштейна и других ученых [5]. Они подчеркивали особый тип расстройств ВНД при эпилепсии, отмечая ослабление памяти, внимания, замедленность мыслительных процессов, нарушения речи и изменения личности. После появления работ У. Пенфилда и Б. Милнер [1-3] большая роль отводилась нейропсихологической диагностике состояния высших психических функций с уточнением роли эпилептического очага. По данным Л.И. Вассермана [6], нейропсихологические заключения о преимущественной стороне поражения совпадали с неврологическими (в 59\% случаев) и психопатологическими (в 71\%), с патологическими отклонениями при электроэнцефалографии (ЭЭГ) 
(в 80-83\%), данными компьютерной томографии - КТ (в 87\%). Некоторые авторы указывают на взаимосвязь коры больших полушарий с лимбическими и стволовыми структурами как на значимый фактор патоморфоза заболевания и различных нарушений ВНД при эпилепсии [2].

В последние десятилетия отмечается, что, помимо грубых нарушений в виде эпилептических психозов и эпилептической деменции, часто наблюдаются изолированные или сочетанные нарушения отдельных функций ВНД, которые тесно связаны с изменениями коры больших полушарий головного мозга разной степени выраженности и в ряде случаев имеют преходящий характер [8].

В развитии когнитивных нарушений важна роль самого эпилептического приступа как проявления заболевания. Выделяют иктальные (приступные) и интериктальные (межприступные) нарушения. Имеются описания возникновения брадипсихии в продромальном периоде приступа по результатам видеомониторинга [9]. К приступным изменениям относят иктальную и постиктальную амнезию, на которые могут жаловаться пациенты с эпилепсией при сборе анамнеза. Также описаны случаи транзиторной эпилептической амнезии. Она наблюдается всего в 5\% случаев, чаще при височной эпилепсии у мужчин старше 60 лет. Приступы возникают обычно 1 раз в месяц и длятся 30-60 мин. В это время человек полностью утрачивает все виды памяти, а затем ощущает этот период как «выпадение» из жизни [10]. Однако не менее важными являются нарушения ВНД, регистрируемые в межприступный период, которые, по выражению Ж. Айкарди, «часто остаются в тени» [9] и не всегда учитываются при ведении пациента. По современным представлениям межприступные когнитивные нарушения - это сложное взаимодействие биологических и социальных факторов, которые носят вариативный характер и могут иметь некоторые различия в зависимости от выбранных методов исследования.

Значимую роль в развитии нарушений ВНД играют анамнестические факторы эпилепсии [1-4, 9]. Возраст появления эпилептических приступов может быть одним из важных факторов в дальнейшем развитии когнитивных нарушений. Наиболее опасным является детский возраст, когда идет созревание головного мозга [8, 9]. D.S. O'Leary и соавт. [11] отмечали более выраженные отклонения в психической сфере у детей с дебютом заболевания в возрасте до 5 лет и дальнейший прогресс изменений связывали с полиформностью приступов и появлением их новых типов в течение заболевания. Эти наблюдения сопоставимы с данными работы [12], в которой представлены результаты исследования группы взрослых пациентов. Было отмечено существенное различие в когнитивной сфере у больных с дебютом эпилепсии до 5 лет и с более поздним дебютом. При дебюте болезни в 14-15 лет и после 18 лет ассоциации с когнитивными нарушениями не выявлено $[13,14]$. Исследователи отмечают, что раннее начало эпилепсии может также обусловливать низкие интеллектуальные способности.

Продолжительность заболевания в большей части исследований [11-14] не являлась специфическим фактором развития нарушений памяти. Ряд авторов показал, что при височной эпилепсии [15] существует связь между продолжительностью заболевания и когнитивными нарушениями. В некоторых случаях при первично-генерализованной эпилепсии когнитивные нарушения связаны с длительностью заболевания, однако корреляция наблюдается у детей в возрасте до 11 лет [16]. Многие исследователи связывают худшие когнитивные способности с частотой приступов $[8,10]$. У монозиготных близнецов с одинаковыми формами эпилепсии, но разной частотой приступов при более частых приступах обнаружено развитие когнитивных нарушений [12].

Влияние половой принадлежности на когнитивные нарушения при эпилепсии до конца не выяснено. Некоторые исследователи отмечают повышение риска развития когнитивных нарушений у мужчин с эпилепсией. Четких патофизиологических объяснений этого нет, однако, по мнению M.L. Smith и соавт. [17], при выполнении тестов на вербальную память и оценке результатов функциональной магнитно-резонансной томографии (МРТ) на определенных этапах кодирования и распознавания информации у женщин левое полушарие активизировалось в большей степени, чем у мужчин.

Характерные различия в когнитивном статусе связаны и с формой эпилепсии [1-10]. Среди фокальных неокортикальных эпилепсий большая доля приходится на височную эпилепсию, при которой чаще всего страдают консолидация памяти, хранение и воспроизведение информации. При левостороннем височном фокусе нарушается вербальная память, возможно развитие сенсорной афазии, тогда как при правостороннем височном фокусе отмечается нарушение пространственной и зрительной памяти. Нередко у пациентов с височной эпилепсией возникают речевые нарушения, но афазия наблюдается нечасто, в основном преобладают бедный лексический запас, трудности с подбором слов.

B. Hermann и соавт. [18] попытались выделить фенотипы когнитивных нарушений при височной эпилепсии, исследовав большую выборку, включавшую 96 пациентов в возрасте от 14 до 59 лет. В зависимости от тяжести нейропсихологических нарушений при височной эпилепсии выделено 3 группы пациентов. В 1-й группе (47\% всех наблюдений) отмечались минимальные когнитивные расстройства в виде статистически значимых по сравнению со здоровыми нарушений памяти, исполнительных функций и речи. У пациентов 2-й группы (24\% больных) обнаружено значительное снижение интеллекта и памяти, исполнительных функций. В 3-й группе (29\% больных) зарегистрированы тяжелые когнитивные отклонения со статистически значимыми изменениями всех сфер когнитивных функций по сравнению с другими группами. Пациенты 3-й группы были старше, имели большую продолжительность заболевания и принимали большее количество противоэпилептических препаратов. В дальнейшем им проведено МРТ-исследование и обнаружена корреляция морфологических изменений височных долей с увеличением тяжести когнитивных нарушений [19].

Рядом исследователей показано, что атрофия левого гиппокампа сочеталась со снижением уровня вербального обучения и памяти, а правого гиппокампа - с дефицитом невербального обучения и памяти [20]. Был сделан вывод, что изменения памяти более очевидны при атрофии левого гиппокампа. По современным представлениям [21], изменение нейрональной пластичности в гиппокампе способствует длительному поддержанию судорожной активности с последующей генерацией судорог. Эти процессы происходят в структурах мозга, отвечающих за выполнение интегративных и мнемонических функций, и влияют на когнитивные процессы. Поэтому представ- 
ляет интерес дальнейшее изучение повреждающего действия эпилептической активности в эпилептогенной или ирритативной зоне коры.

При лобной эпилепсии, как правило, отсутствуют тяжелые нарушения памяти, которые наблюдаются у пациентов с височной эпилепсей [1-7]. Тестируя преиктальный и постиктальный когнитивный профиль, M. Hernandez и соавт. [22] не выявили нарушений вербальной памяти у пациентов с лобной эпилепсией. Эти же авторы показали, что у детей в большей степени страдает поддержание внимания, снижено внимание на зрительные и слуховые стимулы. Обнаруживают также вязкость мышления, поведенческие проблемы, нарушение социализации.

Особые изменения когнитивной сферы происходят при ювенильной миоклонической эпилепсии (ЮМЭ), что, возможно, связано с лобной дисфункцией. По мнению G. Rubboli и C.A. Tassinari [23], эпилептиформная активность и когнитивные изменения при ЮМЭ имеют сложную реципрокную связь. С одной стороны, интериктальные эпилептиформные изменения способны снижать когнитивные способности при этой форме эпилепсии, а с другой сами когнитивные задачи, ставящиеся перед пациентом, могут провоцировать эпилептиформные изменения при ЭЭГ. По сравнению со здоровыми добровольцами у пациентов с ЮМЭ обнаружены нарушения планирования и организации, снижение скорости мышления. В исследовании М.J. Коерр и соавт. [24] при ЮМЭ объем лобных долей не коррелировал с нарушением исполнительных функций, однако дефицит этих функций коррелировал с атрофией таламуса и хвостатого ядра. Данные нейровизуализации представляются интересными в свете роли таламуса, так как ряд авторов [25, 26] предполагает возникновение патологического взаимодействия ретикулярных и релейных ядер таламуса с неокортикальными пирамидными нейронами при некоторых формах генерализованной эпилепсии.

Дополнительная когнитивная нагрузка может провоцировать эпилептиформные изменения, регистрируемые при ЭЭГ. S. Ebus и соавт. [27] регистрировали при появлении интериктальной эпилептиформной активности (ИЭА) широкий спектр когнитивных изменений, но в большей степени снижение скорости обработки информации. Отмечена важность латерализации межприступных эпилептиформных изменений [20]. При регистрации их в левом полушарии наблюдаются речевые нарушения; эпилептиформная активность в правом полушарии или изолированно в правой затылочной области сопровождается визуальнопространственными нарушениями. У детей большое значение имеет частота межприступных эпилептиформных изменений; при анализе 24-часовых амбулаторных ЭЭГ снижение скорости обработки информации и кратковременной памяти выявлялось при наличии ИЭА более чем в $10 \%$ записи бодрствования [27].

Выраженные когнитивные нарушения в сочетании с агрессивными, стойкими изменениями на ЭЭГ при наличии или отсутствии эпилептических приступов в младенческом и детском возрасте обозначаются как «эпилептическая энцефалопатия» [13]. Концепция эпилептических энцефалопатий основана на предположении, что агрессивная иктальная активность в период созревания мозга является главной причинной прогрессирующего когнитивного и нейропсихологического ухудшения или регрес- са. В.А. Карлов [2] также отмечает, что это - состояние, при котором персистирующая эпилептиформная активность сама по себе может вызывать прогрессирующее ухудшение неврологических и психических функций. G.M. Overvliet и соавт. [28] обнаружили у детей с регрессивным аутизмом и когнитивными нарушениями паттерны эпилептиформной активности, схожие с таковыми Ландау - Клеффнера. Они отметили, что у таких детей часто развивались эпилептические приступы. После начала противоэпилептической терапии наблюдалось улучшение, в частности в речевой сфере.

Определенное значение имеют психосоциальные факторы. Отношение общества, трудности социальной адаптации, необходимость постоянной терапии серьезно влияют на эмоциональный фон пациента. У больных эпилепсией нередко встречаются депрессия и тревожность, что может внести существенный вклад в когнитивные нарушения [29].

На развитие когнитивных нарушении влияет и использование противоэпилептических препаратов (ПЭП). B 1926 г. L. Muskens [1] указывал, что изменения личности могут быть следствием такой терапии, в частности применения барбитуратов. По данным исследований, от 30 до 60\% пациентов рассматривают проблемы с памятью и замедлением мышления как побочный эффект ПЭП, хотя во многих работах показано, что когнитивный дефицит присутствовал и до начала лечения [10]. Примечательно, что экспериментальные данные подчеркивают роль длительной противоэпилептической терапии в нарушении клеточной пролиферации и появлении дендритных изменений в зоне CA1 гиппокампа [30]. В свою очередь это может приводить к нарушениям памяти.

Ведется дискуссия о различиях побочных эффектов старых и новых ПЭП. Предпочтительной в лечении эпилепсии является монотерапия, при которой отмечается меньше таких эффектов [8]. Однако использование даже одного препарата может вызывать нарушение функций ВНД. Есть данные об одинаковом ухудшении когнитивных функций у пациентов, принимавших карбамазепин, фенитоин и фенобарбитал [31]. После приема фенитоина и карбамазепина в течение 1 мес когнитивные функции (внимание, исполнительные функции) ухудшались даже у здоровых добровольцев. В многоцентровом исследовании HOLMFRID [32] при отмене карбамазепина или фенитоина отмечалось улучшение функций ВНД. Результаты оценки дозозависимого эффекта карбамазепина не являлись достоверными, но было показано, что когнитивные нарушения возникают в основном при использовании высоких доз препарата.

При сравнении карбамазепина и габапентина было установлено, что габапентин меньше влияет на функции ВНД. Сравнение ламотриджина или препаратов вальпроевой кислоты и карбамазепина [33] выявило положительное влияние ламотриджина на когнитивные функции и даже улучшение внимания при его использовании. Этот препарат минимально влияет на когнитивную сферу как при политерапии, так и при монотерапии [34].

Особый профиль когнитивных изменений ассоциируется с приемом топирамата. При его применении могут достаточно часто возникать речевые нарушения, расстройства памяти и внимания [35].

При сравнении леветирацетама и карбамазепина леветирацетам оказывал меньшее действие на ВНД, в том 
числе на память [36]. Сравнение леветирацетама с плацебо также показало отсутствие негативного влияния на когнитивный профиль.

Таким образом, изучение нарушений функций ВНД при эпилепсии остается актуальной проблемой современной неврологии. Возникновение когнитивных нарушений является неотъемлемой составляющей течения эпилепсии и отражается на общем состоянии пациента в разные стадии развития заболевания. Однако даже современная классификация Международной противоэпилептической лиги (2001) не учитывает в полной мере нарушения функций ВНД.

Достижение ремиссии и контроль побочных эффектов ПЭП - приоритетная задача невролога. Вместе с тем важны социальная адаптация пациента и коррекция возможных когнитивных нарушений. Особенности проявлений и течения заболевания сформировали в обществе негативное мнение об эпилепсии.Такие пациенты испытывают трудности при получении образования и трудоустройстве, создании семьи, у них снижен уровень социального функционирования, что существенно ухудшает качество жизни.

В настоящее время продолжается дискуссия о роли каждого фактора в развитии когнитивных нарушений при эпилепсии, предпринимаются попытки найти оптимальное соотношение доз ПЭП, которые бы не оказывали выраженного влияния на ВНД, изучаются дополнительные методы терапии этих расстройств. Социализация пациентов и помощь при когнитивных нарушениях остаются важной задачей.

\section{Л И T E P A T V $\mathbb{P} A$}

1. Киссин МЯ. Клиническая эпилептология. Москва: Гэотар-Медиа; 2009. [Kissin MYa. Klinicheskaya epileptologiya. Moscow: Geotar-Media; 2009.]

2. Карлов ВА. Эпилепсия у детей и взрослых, женщин и мужчин. Руководство для врачей. Москва: Медицина; 2010.

[Karlov VA. Epilepsiya u detey i vzroslykh, zhenshchin i muzhchin. Rukovodstvo dlya vrachey. Moscow: Meditsina; 2010.]

3. Петрухин АС, Мухин КЮ, Алиханов АА.

Эпилепсия: медико-социальные аспекты.

Москва; 2003. С. 43-64. [Petrukhin AS,

Mukhin KYu, Alikhanov AA. Epilepsiya: mediko-sotsial'nye aspekty. Moscow; 2003. P. 43-64.]

4. Калинин ВВ, Железнова ЕВ, Земляная АА Когнитивные нарушения при эпилепсии. Журнал неврологии и психиатрии им. С.С. Корсакова. 2006;1(106 Приложение Эпилепсия):64-70. [Kalinin VV, Zheleznova EV, Zemlyanaya AA. Kognitivnye narusheniya pri epilepsii. Neuroscience and Behavioral Physiology. 2006;1(106 Prilozhenie Epilepsiya):64-70.]

5. Казаковцев БА. Психические расстройства при эпилепсии. Москва; 1999. С. 416.

[Kazakovtsev BA. Psikhicheskie rasstroystva pri epilepsii. Moscow; 1999. P. 416.]

6. Вассерман ЛИ. Психологическая диагностика при эпилепсии. Русский медицинский журнал. 1998;1(5):1-7. [Vasserman LI. Psikhologicheskaya diagnostika pri epilepsii. Russkiy meditsinskiy zhurnal. 1998;1(5):1-7.]

7. Вассерман ЛИ, Михайлов ВА, Табулина СД Психологическая структура качества жизни больных эпилепсией. Пособие для врачей. Санкт-Петербург: Психоневрологический институт им. В.М. Бехтерева; 2008. С. 22-5. [Vasserman LI, Mikhaylov VA, Tabulina SD. Psikhologicheskaya struktura kachestva zhizni bol'nykh epilepsiey. Posobie dlya vrachey. Sankt-Peterburg: Psikhonevrologicheskiy institut im. V.M. Bekhtereva; 2008. P. 22-5.]

8. Воронкова КВ. Фокальные формы эпи- лепсии у детей: интеллектуальное развитие и варианты нарушений высших психических функций. Дис. ... канд. мед. наук. Москва; 2002. [Voronkova KV. Fokal'nye formy epilepsii u detey: intellektual'noe razvitie i varianty narusheniy vysshikh psikhicheskikh funktsiy. Dis. ... kand. med. nauk. Moscow; 2002.] 9. Петрухин АС. Нейропсихиатрические проблемы эпилепсии. Неврологический журнал. 1999;6:4-10. [Petrukhin AS.

Neyropsikhiatricheskie problemy epilepsii. Nevrologicheskiy zhurnal. 1999;6:4-10.]

10. Броди М. Течение и рациональная терапия эпилепсии. Журнал неврологии и психиатрии им. С.С. Корсакова.

2005;105(10):62-8. [Brodie M. Natural history and rational treatment of epilepsy.

Neuroscience and Behavioral Physiology.

2005;105(10):62-8.]

11. O'Leary DS, Lovell MR, Sackellares JC

et al. Effects of age of onset of partial and generalized seizures on neuropsychological performance in children. J Nerv Ment Dis. 1983 Oct;171(10):624-9.

12. Holmes G. Epilepsy in the developing brain lessons from the laboratory and clinic. Epilepsia. 1997;38(1):12-30. DOI:

http://dx.doi.org/10.1111\%2Fj.15281157.1997.tb01074.x.

13. Карлов ВА. Развивающийся, инволюционирующий мозг, цереброваскулярные заболевания и эпилепсия. Журнал неврологии и психиатрии им. С.С. Корсакова.

2009;109(3):4-7. [Developing brain, involutional brain, cerebrovascular diseases and epilepsy. Karlov VA. Neuroscience and Behavioral Physiology. 2009;109(3):4-7.] 14. Dodrill CB, Troupin AS. Seizures and adaptive abilities. Arch Neurol. 1976;33(9):604-7. DOI:

http://dx.doi.org/10.1001\%2Farchneur.1976.00 500090010003 .

15. Oddo S, Solis P, Consalvo D et al. Mesial temporal lobe epilepsy and hippocampal sclerosis: cognitive function assessment in Hispanic patients. Epilepsy Behav. 2003

Dec;4(6):717-22. DOI:

http://dx.doi.org/10.1016\%2Fj.yebeh. 2006.04.015.

16. Dikmen S, Matthews CG. Effect of major motor seizure frequency upon cognitive-intellectual functions in adults. Epilepsia. 1977

Mar;18(1):21-9. DOI:

http://dx.doi.org/10.1111\%2Fj.1528-

1157.1977.tb05583.x.

17. Smith ML, Elliott I, Naguiat A. Sex differences in episodic memory among children with intractable epilepsy. Epilepsy Behav. 2009

Jan;14(1):247-9. DOI:

http://dx.doi.org/10.1016\%2Fj.yebeh. 2008.08.010

18. Hermann B, Seidenberg M, Lee EJ et al. Cognitive phenotypes in temporal lobe epilepsy. J Int Neuropsychol Soc. 2007 Jan;13(1):12-20. DOI:

http://dx.doi.org/10.1017\%2FS1355617707 07004X.

19. Dabbs K, Becker T, Jones J et al. Brain structure and aging in chronic temporal lobe epilepsy. Epilepsia. 2012 Jun;53(6):1033-43. DOI: http://dx.doi.org/10.1111\%2Fj.15281167.2012.03447.x.

20. Alessio A, Kobayashi E, Damasceno BP et al. Evidence of memory impairment in asymptomatic individuals with hippocampal atrophy. Epilepsy Behav. 2004 Dec;5(6):981-7. DOI: http://dx.doi.org/10.1016\%2Fj.yebeh. 2004.08.018.

21. Гуляева НВ, Ерин АН. Роль свободнорадикальных процессов в развитии нейродегенеративных заболеваний. Нейрохимия. 1995;12(2):3-15.

[Gulyaeva NV, Erin AN. Rol' svobodnoradikal'nykh protsessov v razvitii neyrodegenerativnykh zabolevaniy. Neyrokhimiya.

1995;12(2):3-15.]

22. Hernandez M, Sauerwein HC, Jambaque I et al. Attention, memory, and behavioral adjustment in children with frontal lobe epilepsy. Epilepsy Behav. 2003;4(5):522-36. DOI: 
http://dx.doi.org/10.1016\%2Fj.y

ebeh.2003.07.014.

23. Rubboli G, Tassinari CA. Negative myoclonus. An overview of its clinical features, pathophysiological mechanisms, and management. Neurophysiol Clin.

2006;36(5-6):337-43. Epub 2007 Jan 23. DOI: http://dx.doi.org/10.1016\%2Fj.neu-

cli.2006.12.001

24. Koepp MJ, Woermann F, Savic I,

Wandschneider B. Juvenile myoclonic epilepsyneuroimaging findings. Epilepsy Behav. 2013;28

Suppl 1:S40-4. DOI:

http://dx.doi.org/10.1016\%2Fj.yebeh.2012.06.035.

25. Pulsipher DT, Seidenberg M, Guidotti L

et al. Thalamofrontal circuitry and executive dysfunction in recent-onset juvenile myoclonic epilepsy. Epilepsia. 2009 May;50(5):1210-9. DOI: http://dx.doi.org/10.1111\%2Fj.15281167.2008.01952.x

26. Kelemen A, Barsi P, Gyorsok Z et al. Thalamic lesion and epilepsy with generalized seizures, ESES and spike-wave paroxysmsreport of three cases. Seizure. 2006 Sep;15(6):454-8. DOI:

http://dx.doi.org/10.1016\%2Fj.seizure. 2006.05.006.

27. Ebus S, Arends J, Hendriksen J et al. Cognitive effects of interictal epileptiform discharges in children. Eur J Paediatr Neurol. 2012 Nov;16(6):697-706. DOI:
http://dx.doi.org/10.1016\%2Fj.ejpn. 2012.05.010.

28. Overvliet GM, Besseling RM, Vles JS et al. Nocturnal epileptiform EEG discharges, nocturnal epileptic seizures, and language impairments in children: review of the literature.

Epilepsy Behav. 2010 Dec;19(4):550-8. DOI: 10.1016/j.yebeh.2010.09.015.

Epub 2010 Oct 15.

29. Бурд СГ, Ридер ФК, Бадалян ОЛ и др.

Депрессия и эпилепсия: две стороны одной медали. Русский медицинский журнал. 2008;16(12):1653-8. [Burd SG, Rider FK, Badalyan OL i dr. Depressiya i epilepsiya: dve storony odnoy medali. Russkiy meditsinskiy zhurnal. 2008;16(12):1653-8.]

30. Sgobio C, Ghiglieri V, Costa C et al.

Hippocampal synaptic plasticity, memory, and epilepsy: effects of long-term valproic acid treatment. Biol Psychiatry. 2010 Mar 15;67(6):567-74. DOI: http://dx.doi.org/10.1016\%2Fj.biopsych. 2009.11.008.

31. Cavanna AE, Ali F, Rickards HE, McCorry D. Behavioral and cognitive effects of antiepileptic drugs. Discov Med. 2010 Feb;9(45):138-44.

32. Tonnby B, Nilsson HL, Aldenkamp AP et al. Withdrawal of antiepileptic medication in children. Correlation of cognitive function and plasma concentration-the multicentre
«Holmfrid» study. Epilepsy Res. 1994 Oct;19(2):141-52. DOI:

http://dx.doi.org/10.1016\%2F09201211\%2894\%2990024-8.

33. Aldenkamp AP, Baker G. A Systematic Review of the Effects of Lamotrigine on Cognitive Function and Quality of Life. Epilepsy Behav. 2001 Apr;2(2):85-91. DOI: http://dx.doi.org/10.1006\%2Febeh.2001.0168. 34. Власов ПН, Андреева ОВ. Особенности терапии эпилепсии у пациентов среднего и пожилого возраста. Неврология, нейропсихиатрия, психосоматика. 2009;1:54-8.

[Vlasov PN, Andreyeva OV. Therapy for epilepsy in middle-aged and elderly patients.

Nevrologiya, neyropsikhiatriya, psikhosomatika. 2009;1:54-8.]

35. Loring DW, Williamson DJ, Meador KJ et al. Topiramate dose effects on cognition: a randomized double-blind study. Neurology. 2011 Jan 11;76(2):131-7. DOI: http://dx.doi.org/10.1212\%2FWNL.0b013e318 $206 \mathrm{ca} 02$.

36. Levisohn PM, Mintz M, Hunter SJ et al. Neurocognitive effects of adjunctive levetiracetam in children with partial-onset seizures: a randomized, double-blind, placebo-controlled, noninferiority trial. Epilepsia. 2009 Nov;50(11):2377-89. DOI: http://dx.doi.org/10.1111\%2Fj.15281167.2009.02197.x. 